\title{
A unique case of foreign body aspiration and recurrent pneumothorax
}

\author{
Mario Lima, Sara Ugolini, Niel Di Salvo, Michele Libri, Tommaso Gargano, Giovanni Ruggeri \\ Department of Paediatric Surgery, University of Bologna, Sant'Orsola-Malpighi Hospital, Bologna, Italy
}

\begin{abstract}
Paediatric foreign body aspiration can be related to a high morbidity/mortality rate, especially in young children, and pneumothorax can be a severe associated issue. We describe a case of a 13-year-old girl with recurrent pneumothorax in bilateral bullous lung, twice previously treated at our institution by robotassisted bullectomy, and once again showing same signs and symptoms. At conventional thoracoscopy an inorganic foreign body was found to be located pointing upwards the apex. We believe this may have acted as a contributory cause in the persistence of the clinical picture, despite the surgical removal of the underlying pathology.
\end{abstract}

\section{Introduction}

Paediatric Foreign Body Aspiration (FBA) can be related to a high morbidity and mortality rate, especially in young children. ${ }^{1}$ Most common clinical presentations are cough/airway distress, atelectasis or respiratory infections in the following months/years (for unnoticed aspiration events); Moreover, severe consequences such as pneumothorax or pneumomediastinum can be associated issues, though extremely rare. ${ }^{1}$ Unlike adults, there is no specific paediatric guideline for treatment of pneumothorax. ${ }^{2}$ The most frequently offered surgical treatment

\footnotetext{
Correspondence: Sara Ugolini, Department of Pediatric Surgery, University of Bologna, Sant'Orsola-Malpighi Hospital, via Massarenti 11, 40138 Bologna, Italy.

Tel: +393281138888 - Fax: +390512143086

E-mail: s.ugolini@live.it
}

Conflict of interest: the authors declare no potential conflict of interest.

Key words: Thoracoscopy; Robotic surgery; Foreign body; Pneumothorax; Bullous lung.

Received for publication: 7 September 2017

Accepted for publication: 15 November 2017.

This work is licensed under a Creative Commons Attribution NonCommercial 4.0 License (CC BY-NC 4.0).

(C) Copyright M. Lima et al., 2017

Licensee PAGEPress, Italy

La Pediatria Medica e Chirurgica 2017; 39:171

doi:10.4081/pmc.2017.171 for Primary Spontaneous Pneumothorax (PSP), deriving from adult data and surgeons' personal experience, after failure of the first conservative attempt, is bullectomy performed by VideoAssisted Thoracoscopic Surgery (VATS), usually followed by pleurodesis to prevent recurrence. ${ }^{3} \mathrm{Up}$ to date, many surgical procedures, having indication for Minimally Invasive Surgery (MIS), have also been performed by Robotic System and in the last decade this has been applied to paediatric surgery as well. ${ }^{4}$ The greatest advantages of the robotic system are natural $360^{\circ}$ movements which is notably worthwhile when operating within the rigid closed chest. ${ }^{4}$

We describe a case of a 13-year-old girl with a recurrent right-sided pneumothorax in bilateral bullous lung, twice previously treated at our institution by robot-assisted bullectomy and once again showing same signs and symptoms. During thoracoscopic stapling procedure a foreign body (fishing line) was found to be located pointing upwards the apex of the lung.

\section{Case Report}

A 13-year-old girl presented at our Department in November 2016 with sudden chest pain. A massive right-sided pneumothorax was found on plane X-ray. After having placed a thoracostomy tube, a CT scan was performed (Figure 1) and a bullous pulmonary dysplasia was found bilaterally. For persistent air-leak, the patient was considered candidate for a robotic-assisted resection of bullae and blebs of the right apex. After proper informed consent, the patient was enrolled for surgery. Under general anaesthesia, she was placed in left lateral decubitus. We employed two instrument arms and an accessory thoracoscopic port for suction and insertion of cutting sutures. The incision for the camera was placed $3-4 \mathrm{~cm}$ below the tip of scapula $(12 \mathrm{~mm}$ 3-D camera) and the other next incisions were made at the mutual distance of 7-8 cm. By $8-\mathrm{mm}$ trocars, instrument arms were positioned under direct vision. Initial thoracic exploration was conducted and then the dysplastic area resected (Figure 1). At one-month postoperative X-ray control the patient appeared in active improvement, but two days later she presented again with sudden breath shortness and pain and a massive pneumothorax was shown ipsilaterally. A chest tube was inserted in emergency and at CT scanning few residual bullae were found to be the underlying cause. A second robotic wedge resection was conducted following the previous illustrated technique. After analogous treatment protocol and comparable postoperative course, the patient experienced a new episode. By conventional thoracoscopic approach an inorganic foreign body (fishing line) was intraoperatively found to be located pointing upwards to the apex of the lung and removed by gentle traction (Figure 2). After an uncomplicated early postoperative course, the patient was found to be stable at two years postoperative control. 


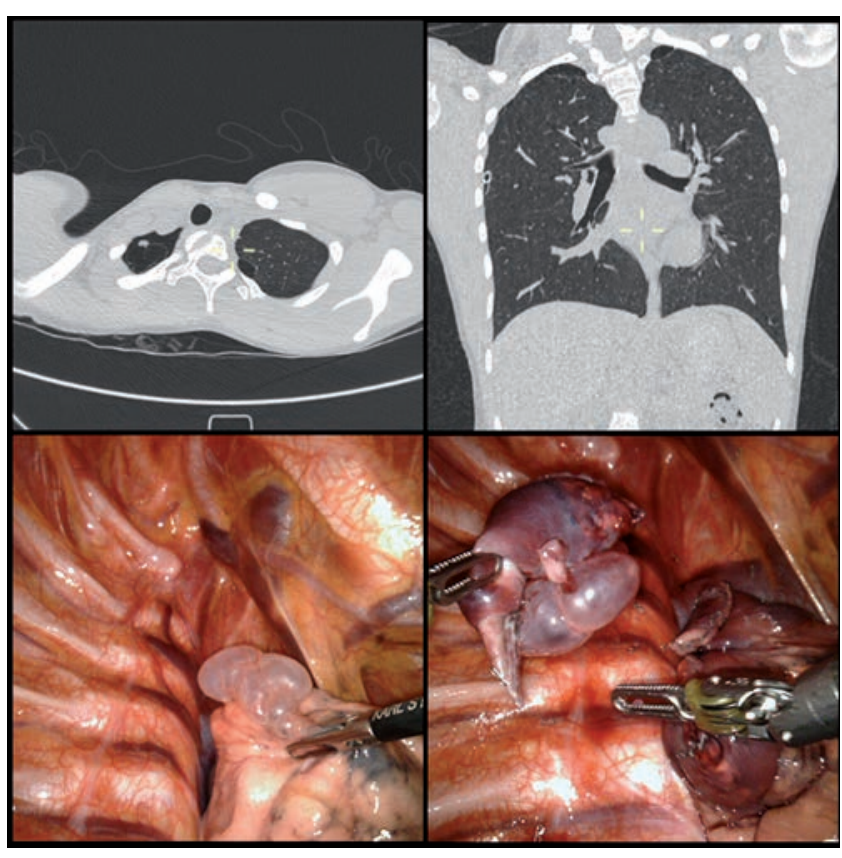

Figure 1. Preoperative computed tomography-scanning, showing pneumothorax and bilateral bullous pulmonary dysplasia, and the robotic-assisted wedge pulmonary resection: first thoracoscopic inspection of bullous right lung, apex resection using the endo-cutting stapler and resected specimen.

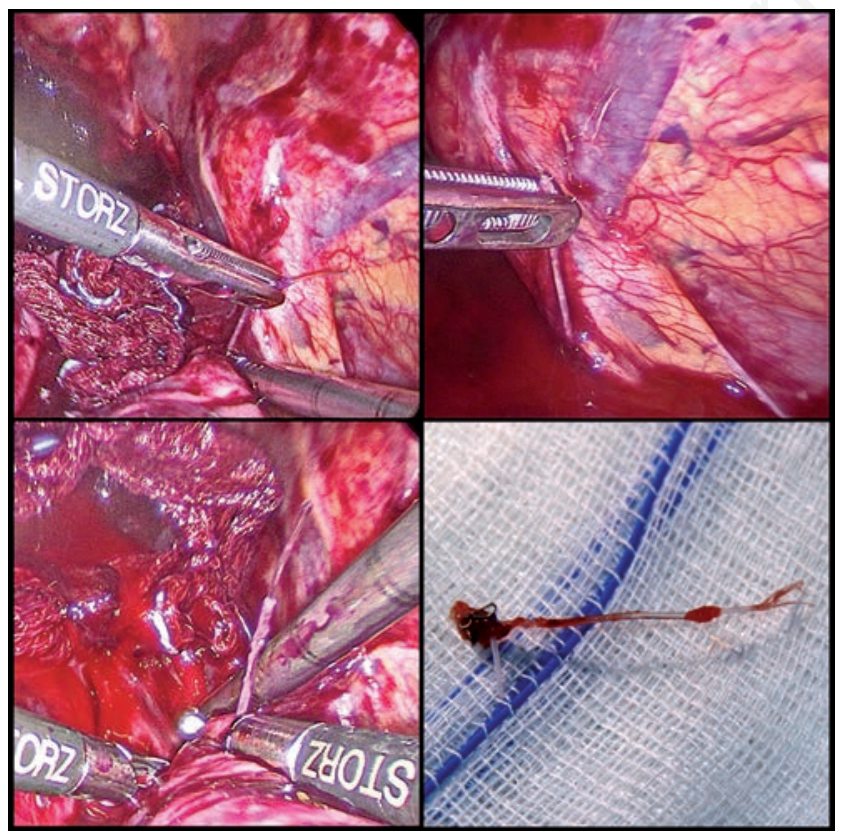

Figure 2. Conventional thoracoscopy redo and intraoperative finding of an inorganic foreign body (fishing line).

\section{Discussion}

Thoracic foreign bodies had been classified into: aspirated, traumatic/accidental or iatrogenic, with the latter reduced to extremely rare events nowadays. Aspirated ones are the most frequent in the paediatric population and clinical consequences reflect sizes, location, and chronicity. When speaking about thoracic surgery in children, patient's size and weight highly influence indications. In fact, the use of the Da Vinci Surgical System, that can be employed to facilitate MIS procedures, remains of limited experience in paediatric age and credit in meaningful series still have to be confirmed.

We presented a case of a 13-year-old girl with bilateral bullous lung and massive right-sided pneumothorax undergoing two robotassisted procedures and one conventional VATS bullectomy due to multiple recurrences. The latter, with the intraoperative finding of an inorganic foreign body (fishing line) that appeared from the parenchymal resection margin (Figure 2). The patient together with parents attributed this to a possible unnoticed aspiration when playing with bracelets years before. No surgical sutures had been utilized in the previously done robotic stapling procedures. Neither similar nor comparable report was found at a literature review.

\section{Conclusions}

We believe the foreign body to be the contributory cause in the perstistence of the clinical picture, despite the surgical removal of the pathological parenchyma. Unlike for adults, there is no specific paediatric guide-line for pneumothorax in children and most frequently applied sur-gical treatment follows adult data and the surgeon's personal experi-ence. 2,3 We decided to treat the patient by means of the removal of the underlying cause (bullae and blebs and pathological parenchy-ma), and no pleurodesis was carried out since long-term effects of these interventions are not known in children. Thoracoscopy in children is reported to be an effective approach with reduced long-term complications compared to thoracotomies (such as chest wall acquired deformities), 5 and, in this case, proved as well very useful in directly recognizing (Figure 2) a foreign body that had been missed on CTs.

\section{References}

1. Gang W, Zhengxia P, Hongbo L, et al. Diagnosis and treatment of tracheobronchial foreign bodies in 1024 children. J Pediatr Surg 2012;47:2004-10.

2. Macduff A, Arnold A, Harvey J. Management of spontaneous pneumothorax: British Thoracic Society pleural disease guideline 2010. Thorax 2010;65(Suppl.2):18-31.

3. Robinson PD, Cooper P, Ranganathan SC. Evidence-based management of paediatric primary spontaneous pneumothorax. Paed Respir Rev 2009;10:110-7.

4. Meehan JJ, Sandler A. Pediatric robotic surgery: a single-institutional review of the first 100 cases. Surg Endosc 2008; 22:177-82.

5. Karpelowsky J. Paediatric thoracoscopic surgery. Paed Respir Rev 2012;13:244-51. 